\title{
Design and Experimental Assessment of a 2D Microwave Imaging System for Brain Stroke Monitoring
}

\author{
Jorge A. Tobon Vasquez, ${ }^{1}$ Rosa Scapaticci ${ }^{\circ},{ }^{2}$ Giovanna Turvani, ${ }^{1}$ Gennaro Bellizzi, ${ }^{3}$ \\ Nadine Joachimowicz, ${ }^{4}$ Bernard Duchêne, ${ }^{5}$ Enrico Tedeschi, ${ }^{6}$ Mario R. Casu ${ }^{1},{ }^{1}$ \\ Lorenzo Crocco $\mathbb{D}^{2}$, and Francesca Vipiana $\mathbb{1}^{1}$ \\ ${ }^{1}$ Department of Electronics and Telecommunications, Politecnico di Torino, 10129 Torino, Italy \\ ${ }^{2}$ Institute for the Electromagnetic Sensing of the Environment, National Research Council of Italy, 80124 Naples, Italy \\ ${ }^{3}$ Department of Electric Engineering and Information Technologies, University of Naples Federico II, 80125 Naples, Italy \\ ${ }^{4}$ Group of Electrical Engineering-Paris (GeePs: CNRS-CentraleSupélec, Univ Paris-Sud, Université Paris-Saclay, \\ Sorbonne Université), 91190 Gif-sur-Yvette, France \\ ${ }^{5}$ Laboratoire des Signaux et Systèmes (L2S: CNRS-CentraleSupélec-Univ Paris-Sud), 91190 Gif-sur-Yvette, France \\ ${ }^{6}$ Department of Advanced Biomedical Sciences, University of Naples Federico II, 80131 Napoli, Italy \\ Correspondence should be addressed to Lorenzo Crocco; crocco.l@irea.cnr.it
}

Received 6 December 2018; Accepted 19 February 2019; Published 5 May 2019

Guest Editor: Alvaro Rocha

Copyright ( 2019 Jorge A. Tobon Vasquez et al. This is an open access article distributed under the Creative Commons Attribution License, which permits unrestricted use, distribution, and reproduction in any medium, provided the original work is properly cited.

\begin{abstract}
The aim of this paper is to present and experimentally verify the first prototype of a microwave imaging system specifically designed and realized for the continuous monitoring of patients affected by brain stroke, immediately after its onset and diagnosis. The device is a $2 \mathrm{D}$ version of the $3 \mathrm{D}$ system, currently under construction, and consists of an array of 12 printed monopole antennas connected to a two-port vector network analyzer through a switching matrix so that each antenna can act as a transmitter or receiver, thereby allowing the acquisition of the entire multistatic multiview scattering matrix required for the imaging. The system has been experimentally tested on $2 \mathrm{D}$ phantoms with electric properties mimicking the brain. The presence and the evolution of the stroke have been reproduced by filling a proper cavity in the phantom with a liquid having the electric properties of blood. A differential approach has been adopted by acquiring the scattering matrix before and after the filling of the blood cavity. The so achieved differential dataset has been processed by means of a linear imaging algorithm in order to reconstruct the stroke location and dimension. Moreover, the effect of pre- and postprocessing operations on the measured data is investigated. A good agreement has been obtained between the reconstructions and the actual scenario. As a final remark, it is worth noting that the entire data acquisition and processing are sufficiently fast to allow a real-time monitoring.
\end{abstract}

\section{Introduction}

In recent years, microwave tomography (MWT) has attracted an increasing interest as an alternative diagnostic tool for medical imaging. Among the various applications, diagnoses of breast cancer [1] and brain stroke [2] are certainly the most prominent examples.

Low cost, reduced size of the equipment, and the use of low-intensity and nonionizing radiations make MWT particularly appealing as compared to standard diagnostic tools for brain stroke, such as magnetic resonance imaging (MRI) and $\mathrm{X}$-ray computerized tomography $(\mathrm{CT})$, which are indeed much more expensive, bulky, and harmful for the patient.

Based on such observations, different microwave system prototypes have been proposed for brain stroke diagnostics in the literature. To the best of our knowledge, the most advanced system for brain stroke diagnosis is the one developed at Chalmers University $[3,4]$. In agreement with the fact that microwave technology must be a support and not a substitute for standard diagnostic tools, this system aims 
at classifying stroke to give the clinicians a prompt information on the kind of stroke they are faced with, which is crucial for an early intervention. The advantages of this device are its small size and weight, as well as a short measurement time that allows real-time diagnosis. Its drawback is that the only information provided is the presence and the kind of stroke, while no information is provided on the size and location. Another crucial point is the need to collect a significant amount of training data to make classification reliable.

As far as stroke imaging is concerned, several systems have been proposed, which are at different stages of development [5-8]. In particular, the BRIM G2 developed at EMTensor [5] for brain stroke tomography and the device developed at the University of Queensland, devoted to intracranial hemorrhage imaging $[6,9,10]$, have been both tested on phantoms as well as on some volunteers with encouraging results.

An application which has been to some extent overlooked for which MWT appears to be particularly promising is the continuous monitoring of the time evolution of the stroke growth after its onset [11]. In fact, monitoring the stroke evolution would give clinicians the possibility to promptly set the therapy and verify its effectiveness, aimed at stopping the growth of the stroke and so at reducing the risk of death or permanent injuries [12-14]. However, frequent, bedside monitoring cannot be done with MRI or X-ray CT, because it requires a completely safe, relatively cheap, and not bulky technology, such as MWT. This is because MWT would allow a repeated long-time exposure of the patient and its embedding at the bedside in the early stages of hospitalization.

In [15], the authors of this contribution have presented the design of a system based on a conformal antenna array adapted to the head, specifically meant for the continuous monitoring of patients affected by brain stroke, immediately after its onset. Starting from the guidelines given therein, this paper presents the ongoing work towards the realization of the first complete prototype of such a system. In particular, we present and experimentally test an initial prototype representing a simplified $2 \mathrm{D}$ version of the device.

In such a prototype, the electronics and the antennas are already the ones of the final system, but instead of a conformal array, a simple circular array of 12 printed monopole antennas is used to image $2 \mathrm{D}$ cylindrical phantoms. In particular, the array is connected to a two-port vector network analyzer (VNA) through a purposely designed switching matrix, so that each antenna can act as a transmitter or as a receiver, thereby allowing the acquisition of the entire multistatic multiview scattering matrix required for imaging. The antenna array is immersed in a coupling medium contained in a tank, acting as a holder.

This 2D system, for which preliminary results are reported in $[16,17]$, has been experimentally tested on phantoms made of a thin plastic material and consisting of waterproof compartments which can be filled with proper liquid mixtures mimicking the electric properties of the different head tissues. The presence and the evolution of the stroke have been reproduced and measured by filling a proper cavity in the phantom with a liquid having the same electric

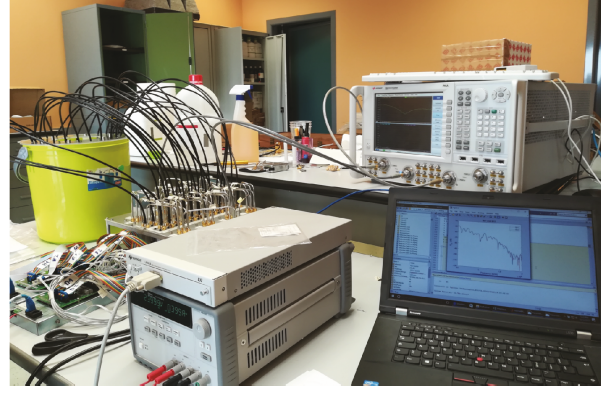

FIgURE 1: Overview of the realized microwave imaging system.

properties than blood and by acquiring the scattering matrix before and after the filling of the cavity. The differential dataset obtained in this way has been then processed by a linear inversion algorithm $[11,18]$ in order to reconstruct the stroke variation. A good agreement has been obtained between the reconstructions and the real positions and sizes assumed for the stroke in the phantom. Moreover, the effect of different pre- and postprocessing operations on the measured data is investigated. The entire data acquisition and processing are sufficiently fast to allow a real-time diagnosis. Notice that it is also possible to accelerate this kind of algorithm with proper specialized hardware, as shown in [19].

The paper is organized as follows. In Section 2, the overall MWT system, including the coupling medium, the antenna array, the switching matrix, the phantom, and the imaging algorithm, is described and characterized. Section 3 describes different pre- and postprocessings applied on the measurement data, in order to assess their influence on the reconstruction. The reconstruction results are reported in Section 4, and conclusion and perspectives follow in Section 5.

\section{Microwave Imaging System}

An overview of the microwave imaging system designed and realized in this work is shown in Figure 1. The system consists of a set of 12 or 24 antennas, placed around the considered phantom, inside a tank filled with the chosen coupling liquid, as shown in Figure 2 in the case of 12 antennas. To act as transmitters and receivers (RX/TX), the antennas are connected to a custom $24 \times 2$ switching matrix, then connected to a vector network analyzer (VNA). Finally, all the signals received by the VNA are used as input data for the implemented imaging algorithm. In the following subsections, all the different parts of the microwave imaging system are detailed, together with the main design constraints.

2.1. Frequency Range and Coupling Liquid. The choice of the working frequency range and the dielectric characteristics of the coupling medium are strictly related to the final use of the realized microwave imaging system, i.e., brain stroke monitoring. According to previous theoretical studies $[20,21]$, the transmission coefficient through a layered medium representing the different tissues of the brain exhibits a "forbidden" band between around $1.5 \mathrm{GHz}$ and $2.5 \mathrm{GHz}$. The frequency behavior of the transmission coefficient depends slightly on the dielectric characteristics of the 


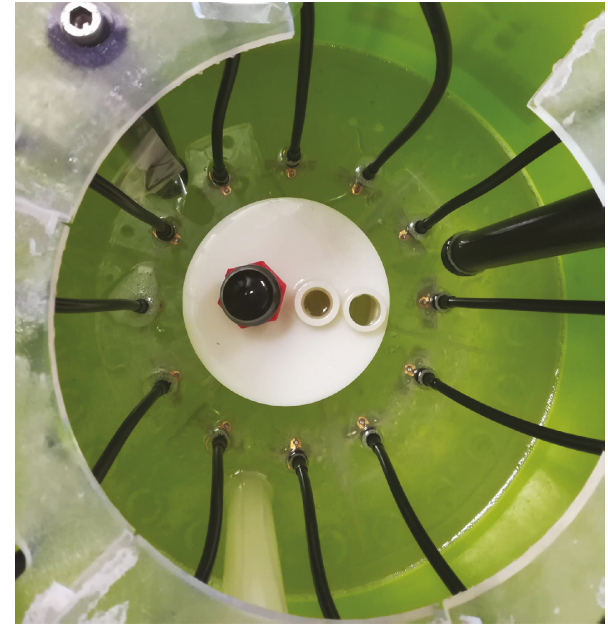

FIGURE 2: Microwave imaging system: tank filled with the coupling liquid with phantom and RX/TX antennas inside.

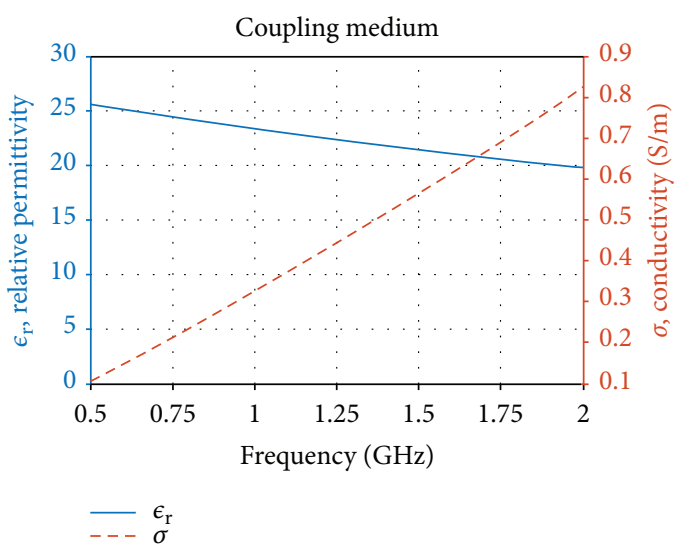

FIGURE 3: Microwave imaging system: coupling medium relative permittivity and conductivity.

coupling medium; in particular, the starting frequency of the forbidden band is a bit higher if its relative permittivity is around 20 or lower. Moreover, working at frequencies higher than $2.5 \mathrm{GHz}$ could be possible but is not convenient, due to the low penetration depth in the inspected tissues. Hence, the chosen working frequency range for the antenna design and image reconstruction algorithms is $1-1.75 \mathrm{GHz}$ with a coupling medium relative permittivity of around 20 .

The desired dielectric characteristics of the coupling medium are obtained with a mixture of Triton X-100 and water in the volume percentage of $70 / 30$; the relative permittivity and conductivity, measured in the working frequency range by means of an open-ended coaxial probe connected to the VNA, are shown in Figure 3. We have chosen a mixture of Triton X-100 and water mainly because it has been verified to be very stable in time and easy to prepare (the two liquids are simply mixed together at room temperature) [22-24]. Moreover, the corresponding attenuation in the considered frequency band (see the conductivity behavior in Figure 3) is much lower than other mixtures proposed in

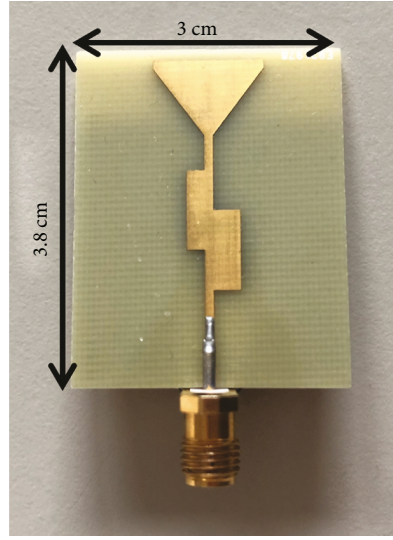

(a)

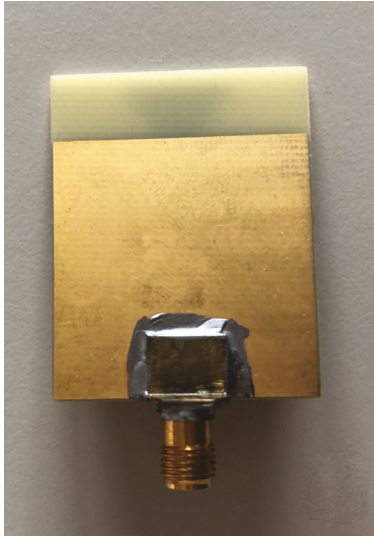

(b)
FIgURE 4: Microwave imaging system: RX/TX antennas; (a): top side; (b): bottom side.

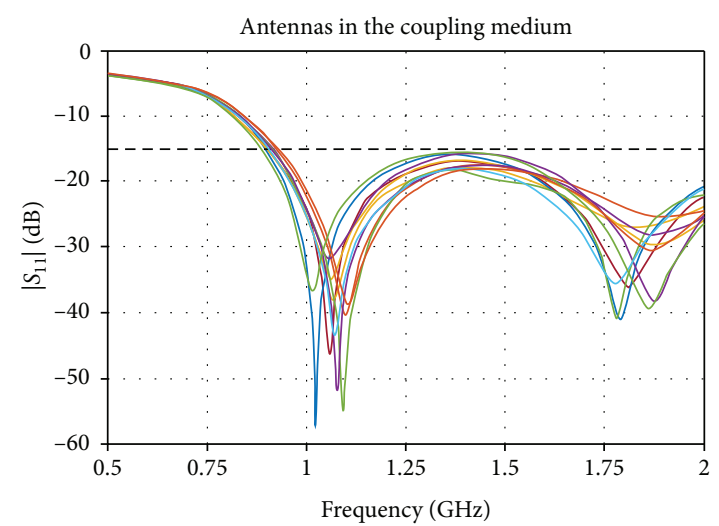

FIGURE 5: Microwave imaging system: amplitude of the input reflection coefficient of the antennas immersed in the coupling medium.

literature, such as the ones based on glycerine [25], allowing to work with less noisy measurements.

2.2. Antennas. The radiating part of the realized microwave imaging system is a set of antennas placed on a circumference, around the considered phantom, and immersed in the coupling medium. The antenna support, inside the tank, allows us to place 24 or 12 antennas on a circumference with a diameter of $24 \mathrm{~cm}$ or $16 \mathrm{~cm}$, respectively, with a distance between adjacent antennas of around $1 \mathrm{~cm}$.

Each antenna of the microwave imaging system is a wideband monopole antenna, printed on a standard FR4 dielectric substrate with thickness equal to $1.6 \mathrm{~mm}$, as shown in Figure 4 . The top side consists of a transmission line with a double-stub matching circuit and ended with a triangularshaped radiating part (see Figure 4(a)). The bottom side is made of a ground plane that terminates close to the beginning of the radiating triangle (see Figure 4(b)).

Figure 5 reports the amplitude of the input reflection coefficient of the realized antennas immersed in the coupling medium: the matching is always below $-15 \mathrm{~dB}$ all over the frequency range $(1-1.75 \mathrm{GHz})$. The differences in 


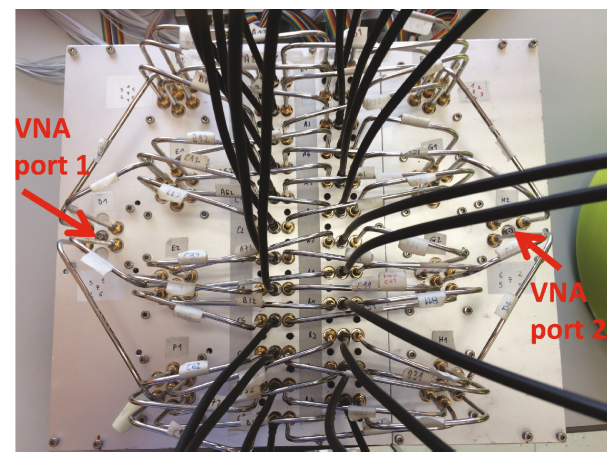

FIGURE 6: Microwave imaging system: switching matrix.

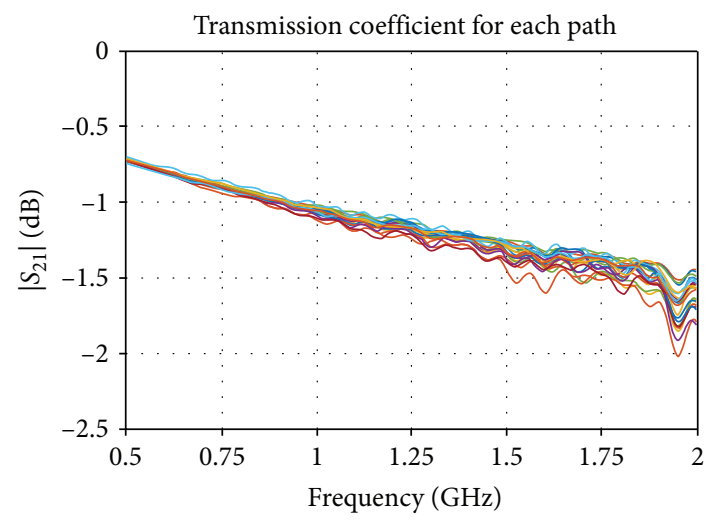

Figure 7: Microwave imaging system: amplitude of the transmission coefficient of each path of the switching matrix.

the behavior of the measured antennas are expected to be related mainly to the slightly different welding of the connector (see Figure 4) and also to the possible presence of air bubbles in the coupling liquid wherein they are immersed. Moreover, the presence of a double resonance at the two limits of the frequency range allows us to work also in an almost "flat" region (around $1.5 \mathrm{GHz}$ ), where the matching is still below $-15 \mathrm{~dB}$ and the $\left|S_{11}\right|$ coefficient differs much less with respect to the resonant regions. This is an interesting feature because it has been verified that the implemented imaging algorithm performs better in this flat region (see Section 4).

The realized antennas are wide-band monopoles, hence with an omnidirectional radiation pattern in the plane orthogonal to the antenna plane (see Figure 4). This plane corresponds to the one where the antennas are placed on a circumference (see Figure 2); hence, a high coupling is expected between adjacent antennas. In order to mitigate this effect, the scattering parameters between adjacent antennas are discarded in the implemented imaging algorithm (see Section 4).

2.3. Switching Matrix. In the microwave imaging system, each antenna acts as a transmitter and as a receiver, in order to have multistatic multiview measurements of the phantom. Hence, all the antennas are connected to the VNA through a switching matrix, shown in Figure 6. The switching matrix consists of two single-pole-four-throw (SP4T), eight single- pole-six-throw (SP6T), and twenty-four single-pole-doublethrow (SPDT) electromechanical coaxial switches. The pole of the two SP4T switches is connected via a flexible coaxial cable to one port of the VNA, and the pole of the twentyfour SPDT switches is connected, in the same way, to one antenna port. The other ports of the switches are properly connected together via semirigid coaxial cables in order to implement a $2 \times 24$ switching matrix. The semirigid coaxial connections among the SP4T, SP6T, and SPDT switches have been realized in order to have very similar path lengths between the VNA port and any antenna port.

To create the proper connections between the antennas and the VNA, all the switches are connected to the control board. The Ethernet connectivity available on the board enables the connection to the network, which allows us to control the entire system from a standard laptop. The board provides a built-in graphical web interface which enables a full manual configuration of the matrix. Moreover, in order to make fast and automated measurements, the switches can also be controlled through the VISA/TCPIP (VXI-11) protocol by using standard scripting languages such as MATLAB. This approach has been followed in the present prototype in order to enable a software integration of the measurement data and the imaging algorithm. In this way, complete automated scans of the target can be run providing the reconstructed images in almost real time. In the current prototype, the measurement time is around three minutes, considering the frequency range $0.5-3 \mathrm{GHz}$ with 1001 sample points and an intermediate-frequency (IF) bandwidth equal to $1 \mathrm{kHz}$, and the processing of the measured data thought the imaging algorithm needs a few seconds.

In the implementation of the switching matrix, we have chosen electromechanical coaxial switches in order to minimize the insertion loss $(<0.3 \mathrm{~dB})$ and maximize the isolation $(>90 \mathrm{~dB}$ ), because it is expected that the useful signal (i.e., the one due to the stroke variation) is typically about $70 \mathrm{~dB}$ below that measured at two monitoring times (which is due to the scattering from the whole head); hence, it is a very critical signal to be detected $[15,26]$. Figure 7 shows the amplitude of the transmission coefficient of each path of the switching matrix from the VNA port to the antenna port, including also the $1 \mathrm{~m}$ flexible coaxial cable connecting the antenna to the matrix; it has been verified that the introduced attenuation is no more than $2 \mathrm{~dB}$ in the considered frequency range.

2.4. 2D Phantom. The experiments presented herein (see Section 4) have been carried out with a 3D-printed phantom $[22,23]$ designed to model the possible presence of a stroke area inside the brain. The phantom, shown in Figure 8, is a $10 \mathrm{~cm}$ diameter, $16 \mathrm{~cm}$ high circular cylindrical cavity made of acrylonitrile butadiene styrene (ABS) and closed by a cap with three holes where smaller ABS cylinders can be inserted; the larger hole has a diameter of $1.8 \mathrm{~cm}$ and the other two have a diameter of $1.4 \mathrm{~cm}$. Note that this phantom is meant to carry out preliminary $2 \mathrm{D}$ measurements and, as such, one dimension (the height) is dominant with respect to the other (diameter) and it does not take into account the actual dimensions of a human head. Of course, the 3D head phantom will be developed with realistic dimensions. 


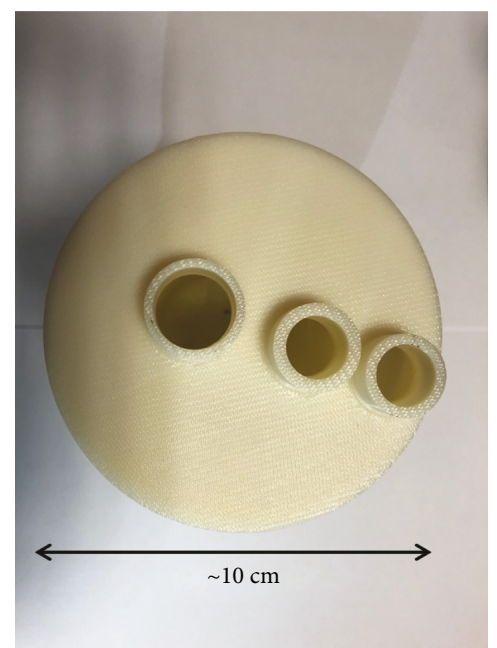

(a)

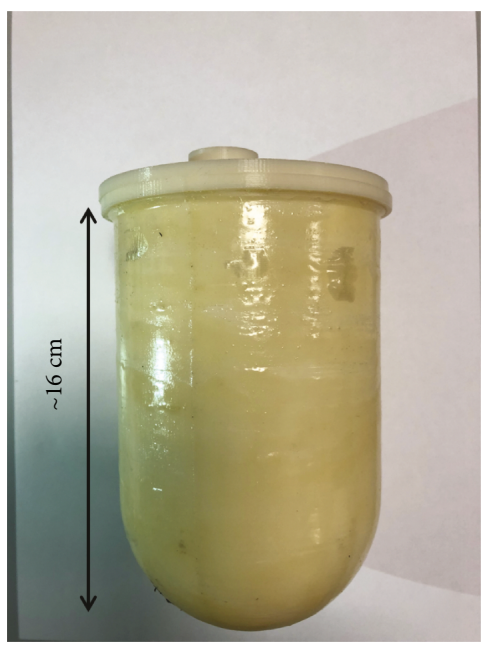

(b)

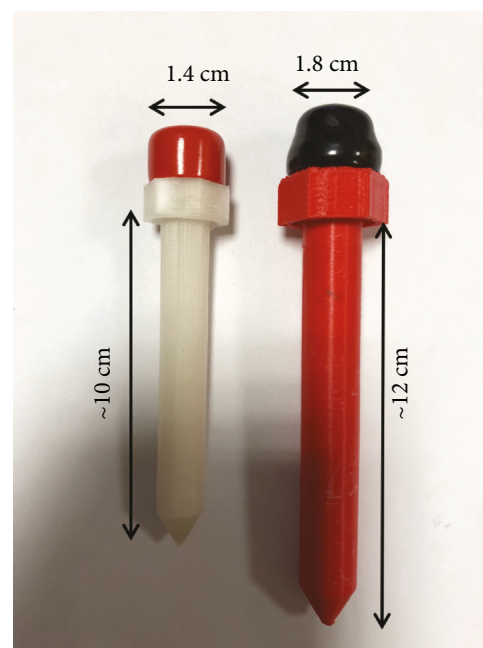

(c)

Figure 8: 2D phantom: (a): top view; (b): side view; (c): inserted cylinders.

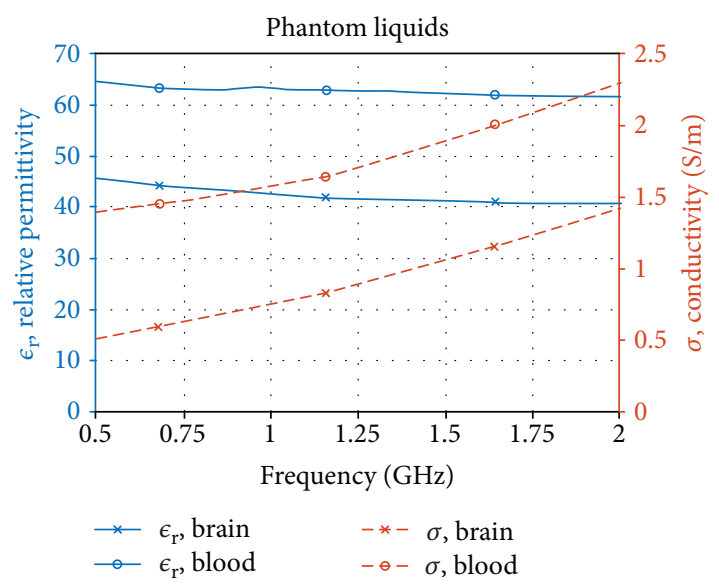

FIGURE 9: 2D phantom: dielectric properties of the liquids used to fill the different parts of the phantom.

As shown in Figure 2, the phantom is immersed in a coupling liquid and placed at the center of the circular antenna support. The distance between the antennas and the phantom external surface is about $3 \mathrm{~cm}$; in the operating frequency range, this corresponds to a distance in between $0.5 \lambda$ and $0.8 \lambda$, where $\lambda$ is the wavelength in the coupling medium. This choice results from a trade-off in minimizing the attenuation of the useful signal on one hand and, on the other hand, the near-field effects that are hard to model and that could perturb the measured fields $[15,27]$. It can be noted that, considering the location of the antennas and the cylindrical shape of the phantom, the latter is assumed to be a 2D target and the images are built up in the antenna support plane, orthogonal to the cylindrical cavity axis (see Section 4).

The phantom cavity is filled up with a liquid that mimics the brain, the latter being modeled as a blend of white and grey matters ( $75 \%$ of white matter and $25 \%$ of grey matter), while the inner cavity is filled up with another mixture representing the blood. The brain- and blood-mimicking mixtures are made of Triton X-100 (TX-100), water, and salt with respective volume fractions and salt quantities of $38 \%$ of TX-100, $72 \%$ of water, and $5.2 \mathrm{~g} / \mathrm{L}$ of salt for the brain and $14 \%$ of TX-100, $86 \%$ of water, and $9.4 \mathrm{~g} / \mathrm{L}$ of salt for the blood [28]. Finally, Figure 9 reports the dielectric properties of the tissue-mimicking mixtures measured by means of an openended coaxial probe connected to the VNA in the 0.5$2 \mathrm{GHz}$ operating frequency range.

2.5. Imaging Algorithm. The data collected with the system are processed by means of a simple and well-assessed approach, based on the singular value decomposition (SVD) of the scattering operator relating the data of the problem to the unknown contrast function [18].

Since the target application of the system at hand is monitoring the time evolution of the stroke after its onset, i.e., the variation of the electric contrast of the brain tissues at two different times, the data of the imaging problem are represented by the difference between the scattering matrices measured at these two different times. This differential scattering matrix will be denoted as $\Delta S$ in the following.

Typically, the differential contrast, say $\Delta \chi$, associated to $\Delta S$ is localized in a small portion of the imaging domain. Accordingly, it is possible to take advantage of the distorted Born approximation, so that $\Delta S$ and $\Delta \chi$ become linearly related through the following relationship:

$$
\Delta S\left(r_{p}, r_{q}\right)=\mathcal{S}(\Delta \chi)
$$

where $\mathcal{S}$ is a linear and compact integral operator, whose kernel is $-j \omega \epsilon_{b} / 4 E_{\mathrm{b}}\left(r_{m}, r_{p}\right) \cdot E_{\mathrm{b}}\left(r_{m}, r_{q}\right)$, with $r_{m} \in D, E_{\mathrm{b}}\left(r_{m}, r_{p}\right)$ being the background field in the unperturbed scenario, while $r_{p}$ and $r_{q}$ denote the positions of the transmitting and receiving antennas, and $r_{m}$ are the positions of the points in which the imaging domain $D$ is discretized. The symbol "." denotes the dot product between vectors, $\omega=2 \pi f$ is the angular frequency, $\epsilon_{b}$ the relative dielectric constant of the background medium, and $j$ the imaginary unit. 


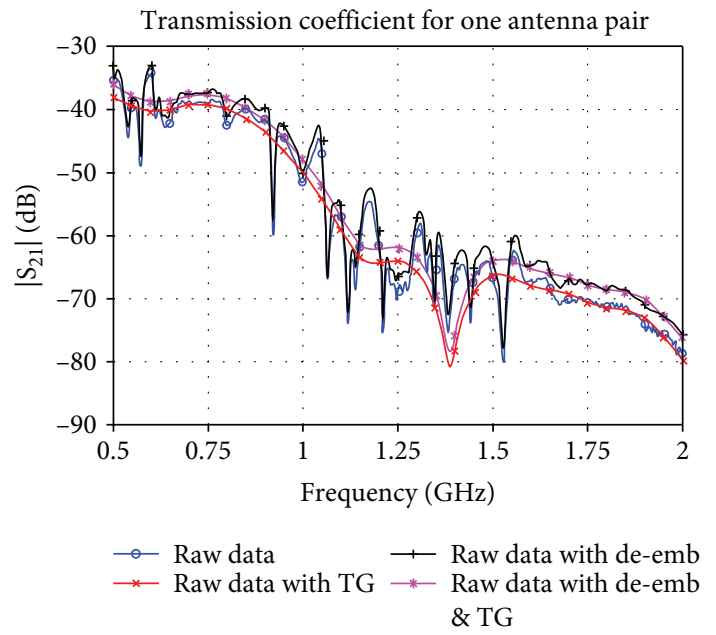

Figure 10: Amplitude in $\mathrm{dB}$ of the transmission coefficient versus frequency for a pair of antennas comparing the raw measured data, the data with time gating (TG), the data with deembedding, and the data with TG and deembedding.

The linear problem in (1) can be inverted by means of the truncated singular value decomposition (TSVD) scheme [18], and the retrieved differential contrast reads

$$
\Delta_{\chi}=\sum_{n=1}^{L_{t}} \frac{1}{\sigma_{n}}\left\langle\Delta S,\left[u_{n}\right]\right\rangle\left[v_{n}\right]
$$

where $\langle[u],[\sigma],[v]\rangle$ is the SVD of the discretized scattering operator $\mathcal{S}$. $L_{t}$ is the truncation index of the SVD, which acts as a regularization parameter.

\section{Pre- and Postmeasurement Processing}

As described in Section 2.3, the VNA is connected to the two input ports of the switching matrix, whose output ports are then connected, via flexible coaxial cables, to the TX/RX antennas. The VNA is a N5227A PNA Microwave Network Analyzer, with an output power of $0 \mathrm{dBm}$ and calibrated with a full two-port (twelve terms) calibration at the end of its high-precision coaxial cables (then connected to the switching matrix), in order to eliminate the directivity error, the cross-talk, the source match error, and the frequency response reflection tracking error.

Figures 10 and 11 show the amplitude and unwrapped phase, respectively, of the measured $S_{21}$ coefficient for one antenna pair of the realized microwave imaging system. The data, labelled as "raw data," are the $S_{21}$ coefficients measured at the calibrated VNA ports, while the "raw data with TG" are the measured data with a time gating applied in order to reduce multiple reflections within the tank. To get the $S_{21}$ coefficient in the time domain, an Inverse Fourier Transform (IFT) is performed on the measured data points, as shown in Figure 12 (solid line). Then, a windowed gating function, centered at $17.5 \mathrm{~ns}$ and with a time span of $9 \mathrm{~ns}$, is applied. The corresponding time-gated data, shown in Figure 12 (dashed line), contain the main impulse received

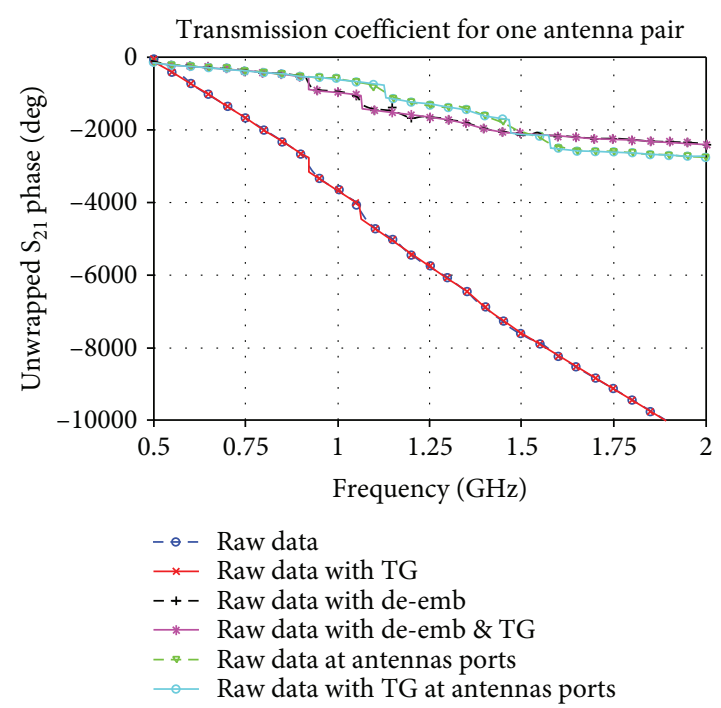

FIgURE 11: Unwrapped phase in degrees of the transmission coefficient versus frequency for a pair of antennas comparing the raw measured data, the data with time gating (TG), the data with deembedding, and the data with TG and deembedding; the raw data measured directly at the antenna ports are shown as reference.

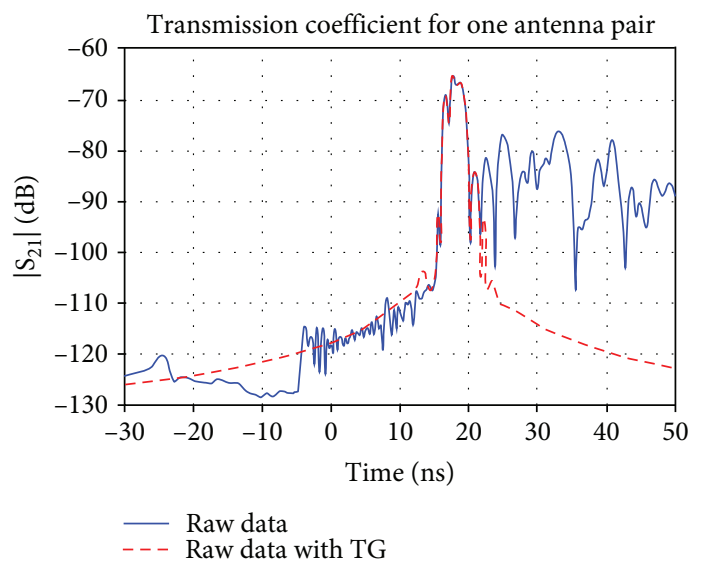

FIgURe 12: Amplitude in $\mathrm{dB}$ of the transmission coefficient versus time for a pair of antennas comparing raw measured data and data with time gating (TG).

by the considered antenna with less reflections from the tank. The gated time domain response is then transformed back to the frequency domain with the effect that most of the multiple reflections are removed, as shown in Figure 10. Comparing the amplitude of the measured $S_{21}$ coefficient in the frequency domain, it is evident that the time-gated data are, as expected, much smoother than the initial data; on the contrary, there are no changes in the measured phase (see Figure 11).

In order to obtain the transmission coefficients directly at the antenna ports for all the considered paths (as expected by most imaging algorithms), a deembedding technique is applied to the measured data. Each measured $2 \times 2[S]$ matrix includes three 2-port parts, each one represented by its own $2 \times 2$ scattering matrix: the input (left) path between the port 1 of the VNA and the TX antenna through the switching 


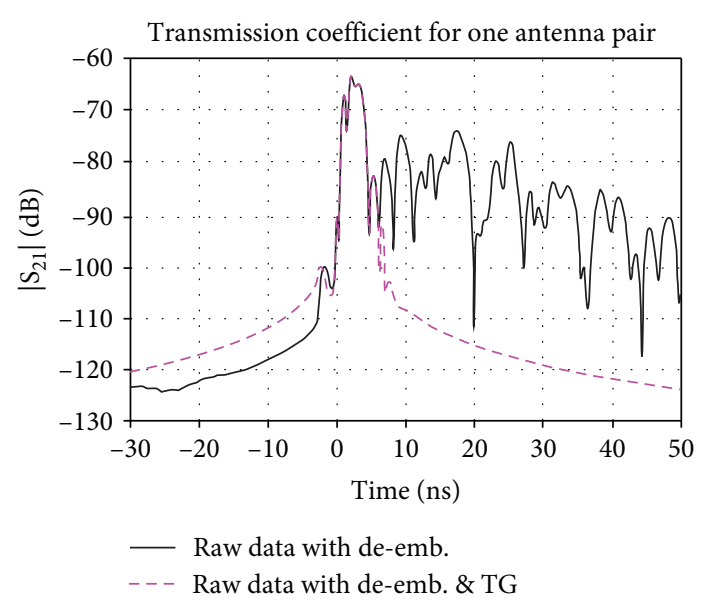

Figure 13: Amplitude in $\mathrm{dB}$ of the transmission coefficient versus time for a pair of antennas comparing deembedded raw measured data and deembedded data with time gating (TG).

matrix represented by $\left[S_{\mathrm{L}}\right]$, the TX/RX antenna pair represented by $\left[S_{\text {DUT }}\right]$, and the output (right) path between the $\mathrm{RX}$ antenna and the port 2 of the VNA via the switching matrix represented by $\left[S_{\mathrm{R}}\right]$. In order to "extract" the desired $\left[S_{\text {DUT }}\right]$, the corresponding $\left[S_{L}\right]$ and $\left[S_{R}\right]$ are measured with the calibrated VNA. Then, all the measured scattering matrices, $[S],\left[S_{L}\right]$, and $\left[S_{R}\right]$, are rewritten into the corresponding transmission matrices, $[T],\left[T_{\mathrm{L}}\right]$, and $\left[T_{\mathrm{R}}\right]$, respectively [29]. Considering that

$$
[T]=\left[T_{\mathrm{L}}\right]\left[T_{\text {DUT }}\right]\left[T_{\mathrm{R}}\right],
$$

where $\left[T_{\mathrm{DUT}}\right]$ is the unknown transmission matrix of the considered TX/RX antenna pair, the $\left[T_{\mathrm{DUT}}\right]$ matrix is obtained as

$$
\left[T_{\text {DUT }}\right]=\left[T_{\mathrm{L}}\right]^{-1}[T]\left[T_{\mathrm{R}}\right]^{-1}
$$

and rewritten as a scattering matrix in $\left[S_{\text {DUT }}\right]$.

For the considered antenna pair, Figures 10 and 11 show the amplitude and unwrapped phase of the measured $S_{21}$ coefficient after the application of the described deembedding (lines labelled as "raw data with de-emb"). We can observe that the amplitude of the $S_{21}$ coefficient, after deembedding, is very similar to the initial one, which just shifted up of around 1.5-4 dB (within the considered frequency range) due to removal of the attenuation through the scattering matrix (see Figure 7, considering two paths). On the contrary, the phase is changed and, as expected, it is superimposed to the one measured directly at the antenna ports (labelled as "raw data at antenna ports" in Figure 11).

As for the previous case, a time gating is then applied to the deembedded data in order to mitigate multiple reflections within the tank. Hence, the deembedded data are transformed in the time domain (see Figure 13, solid line) and a windowed gating function, centered at $2 \mathrm{~ns}$ and with the same time span as before ( $9 \mathrm{~ns})$, is applied. The gating function is now applied at the beginning of the time domain $S_{21}$ coefficient because, due to the deembedding, the reference planes are at the antenna ports. The gated time domain response is then transformed back to the frequency domain, as shown in Figures 10 and 11 (lines labelled as "raw data with de-emb. \& TG").

All the previously described kinds of measured data, with or without time gating and with or without deembedding, are used in Section 4 to generate the images via the implemented imaging algorithm.

\section{Experimental Results and Discussion}

The experimental measurement campaign is reported in this section in order to test the developed system; it has been carried out with the phantom described in Section 2.4 and with the imaging algorithm detailed in Section 2.5.

Three cases are considered according to the available holes in which the inner cylinder, mimicking the hemorrhagic stroke, can be inserted in the phantom, mimicking the brain. In particular, the following three cases are reported:

(i) Case 1: $1.4 \mathrm{~cm}$ cylinder positioned nearby the center of the phantom, centered at -13 and $-7.5 \mathrm{~mm}$

(ii) Case 2: $1.8 \mathrm{~cm}$ cylinder positioned nearby the center of the phantom, centered at 7.5 and $13 \mathrm{~mm}$

(iii) Case 3: $1.4 \mathrm{~cm}$ cylinder positioned close to the periphery of the phantom, centered at -32.5 and $18.75 \mathrm{~mm}$

For each case, the differential measurements have been obtained by subtracting the scattering parameters gathered in the presence of the phantom without the target cylinder. Moreover, to minimize the antenna coupling effects, the nearest antenna contributions are removed from the differential scattering matrix given in input to the reconstruction algorithm.

The performance of the system is analyzed with respect to the working frequency band (within the operative range of the antennas), with respect to the adopted pre- and postmeasurement operations, as discussed in Section 3, and with respect to the knowledge of a priori information on the background scenario, i.e., the scenario with the phantom and without the target cylinder. As far as the frequency range is concerned, with reference to the matching of the antennas summarized in Figure 5, we have identified two possible operative situations: the first range is $1-1.5 \mathrm{GHz}$, including the main resonance of the antennas, and the second one is $1.5-1.75 \mathrm{GHz}$, where the $S_{11}$ coefficient still confirms a good matching, even outside the main resonance. In the following analysis, we refer to low-frequency range when processing multifrequency data in the range $1-1.5 \mathrm{GHz}$, while high-frequency range denotes the processing of multifrequency data in the range $1.5-1.75 \mathrm{GHz}$. Concerning pre- and postmeasurement procedures, we have analyzed all the four possible solutions identified in Section 3, namely, raw data, raw data with time gating, raw data with deembedding, and raw data with deembedding and time gating. Finally, the robustness with respect to a priori knowledge on the reference scenario 
has been evaluated by comparing results obtained with Green's function of the homogeneous coupling medium with and without the presence of the brain phantom. Both Green's functions have been numerically obtained by full-fledged simulations of the system by means of an in-house vectorial 3D finite element method (FEM) solver [15]. Finally, to quantitatively appraise the quality of the reconstructions, three metrics have been defined and evaluated for the considered examples:

(i) Dimensional error (Dim), defined as the ratio between the retrieved dimension of the target (once the image has been binarized through a $-3 \mathrm{~dB}$ thresholding) and the actual dimension of the target

(ii) Localization error (Loc), defined as the distance between the retrieved and the actual centers of the target

(iii) Side lobe level (SLL), defined as the ratio of the highest value of the retrieved differential contrast falling outside the target with respect to the highest value attained inside the target

For the sake of brevity, the complete analysis for Case 1 is reported in the following, while for Case 2 and Case 3, only the best condition results (coming from the previous analysis) are shown.

Figure 14 and Table 1 summarize the analysis of performances in Case 1. First, we can observe that the application of time gating to raw data as well as to deembedded data improves the accuracy of the reconstructions, mainly in terms of side lobe level, even if a reconstruction is feasible also without applying it. On the contrary, the deembedding operation seems to bring only a slight improvement in the reconstructed images, despite the significant improvement of the phase estimation of the measured data (see Figure 11), probably due to the fact that the switching matrix has been built such to keep all the patterns of the same length and hence, the correction of the phase is not significant in the developed system. As far as the frequency range is concerned, from both Figure 14 and Table 1, it can be concluded that the high-frequency ranges are to be preferred. It is worth to note that the thresholds of the singular values for the low- and high-frequency range are different $\left(\tau=20 \log _{10}\left(\sigma_{L t} / \sigma_{1}\right)=-22 \mathrm{~dB}\right.$ for low frequencies and $\tau=$ $-32 \mathrm{~dB}$ for high frequencies). This is in agreement with their decay in the two cases, reported in Figure 15, where it can be seen that at these different threshold values correspond around the same number of singular values (and hence basis functions used in the reconstruction formula in (2)).

As far as it concerns the adopted a priori information on the scenario, the results show that using Green's function that takes into account the presence of the brain phantom does not bring to significant improvements with respect to the simple Green function of the homogeneous coupling medium. This is possibly due to the fact that the features of the phantom (i.e., geometry, electric properties, and position with respect to the antenna array) are not known with sufficient accuracy such to allow a remarkable improvement. As this is even more true in practical applications, we can expect that the TSVD reconstruction of the stroke evolution is not likely to be significantly improved by exploiting patientspecific prior knowledge (e.g., geometry and electric properties of the head).

Given the above analysis, the selected settings for the processing of data related to Case 2 and Case 3 are summarized as follows:

(i) High-frequency range data $(1.5-1.75 \mathrm{GHz})$

(ii) Green's function of the homogeneous coupling medium

(iii) Time-gating measurement data processing

Figures 16 and 17 show the obtained reconstructions, while Table 2 summarizes the quantitative metrics evaluated for these examples. As can be noticed, the $1.8 \mathrm{~cm}$ cylinder is accurately reconstructed. The size and shape of the $1.4 \mathrm{~cm}$ cylinder are also correctly imaged, but the retrieved target position is radially displaced towards the center. Such an outcome could have been to some extent expected, since the $1.4 \mathrm{~cm}$ cylinder is placed close to the phantom boundary, whose effect is not taken into account in the homogeneous Green function. The boundary represents a significant electric discontinuity and therefore introduces a modeling error, especially for targets located in its proximity. On the other hand, given the already mentioned circumstance that the phantom is not known with sufficient precision, no significant improvements are obtained using the more accurate (but still approximated) Green function.

\section{Conclusion and Perspectives}

In this paper, the prototype of a MWT system designed for the continuous imaging of the brain stroke evolution, after its onset, has been presented and experimentally tested. The system is composed of a ring of twelve printed monopole antennas, designed to work in the band $1-1.75 \mathrm{GHz}$; a switching matrix enabling the acquisition of the entire scattering matrix through a two-port VNA; and a coupling medium whose electric properties have been tuned such to enable a good trade-off between wave penetration and attenuation inside the phantom. Moreover, a phantom has been built up, which can be filled with different liquids, mimicking a human brain affected by a hemorrhagic stroke. Each part of the system has been experimentally characterized in order to set the best working frequency and to accurately compute Green's function used in the reconstruction algorithm, which is a linear imaging algorithm based on TSVD. The input differential data, arising from the stroke evolution, have been simulated by measuring the scattering matrix before and after the filling of the cavity of the phantom mimicking the stroke.

Different pre- and postprocessing operations have been applied to the measured differential scattering matrix in order to assess their effect on the quality of the image reconstruction. In particular, in order to reduce the effect of the antenna mutual coupling, in all cases, the scattering 

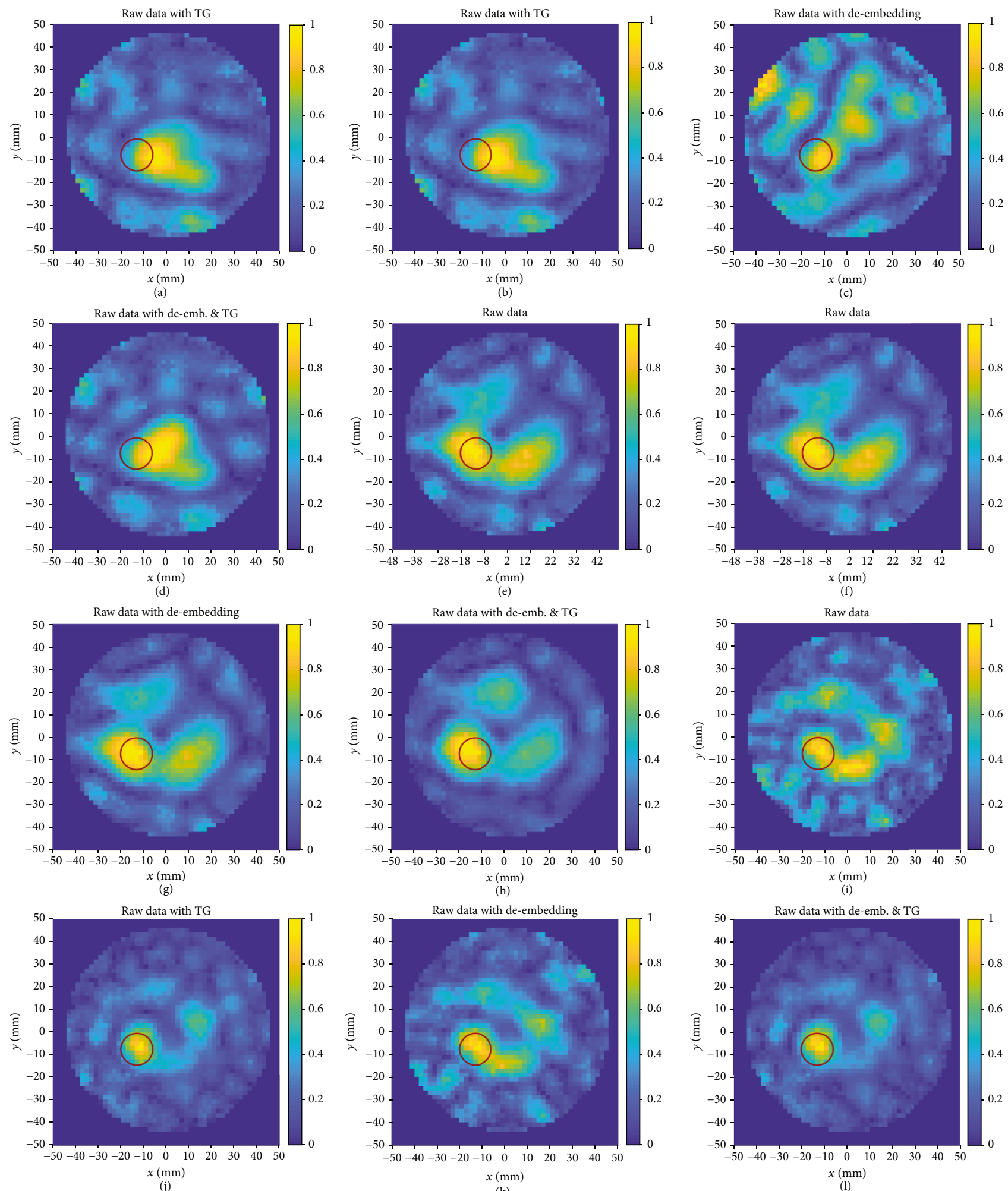

FIGURE 14: Reconstruction images for Case 1: first column: raw data, second column: raw data with time gating (TG), third column: raw data with deembedding, and fourth column: raw data with deembedding and TG; first row: 1, 1.25, and 1.5 GHz with Green's function of the homogeneous coupling medium and $\tau=-22 \mathrm{~dB}$; second row: 1.5 and $1.75 \mathrm{GHz}$ with Green's function of the homogeneous coupling medium and $\tau=-32 \mathrm{~dB}$; third row: 1.5 and $1.75 \mathrm{GHz}$ with Green's function of the homogeneous coupling medium inside the phantom and $\tau=-32 \mathrm{~dB}$. 
TABLE 1: Case 1 evaluated metrics.

\begin{tabular}{|c|c|c|c|c|}
\hline \multicolumn{5}{|c|}{ Green's function of the homogeneous coupling medium } \\
\hline & Raw data at $1-1.5 \mathrm{GHz}$ & TG data at $1-1.5 \mathrm{GHz}$ & De-emb. data at $1-1.5 \mathrm{GHz}$ & TG de-emb. data at $1-1.5 \mathrm{GHz}$ \\
\hline Dim & 0.83 & 1.31 & 0.86 & 1.31 \\
\hline Loc & $3.03 \mathrm{~mm}$ & $4.71 \mathrm{~mm}$ & $2.5 \mathrm{~mm}$ & $6.9 \mathrm{~mm}$ \\
\hline \multirow[t]{2}{*}{ SLL } & $0.88 \mathrm{~dB}$ & $-2.38 \mathrm{~dB}$ & $0.73 \mathrm{~dB}$ & $-3.1 \mathrm{~dB}$ \\
\hline & Raw data at $1.5-1.75 \mathrm{GHz}$ & TG data at $1.5-1.75 \mathrm{GHz}$ & De-emb. data at $1.5-1.75 \mathrm{GHz}$ & TG de-emb. data at $1.5-1.75 \mathrm{GHz}$ \\
\hline Dim & 1.33 & 1.1 & 1.14 & 1 \\
\hline Loc & $2.51 \mathrm{~mm}$ & $2 \mathrm{~mm}$ & $3 \mathrm{~mm}$ & $4.2 \mathrm{~mm}$ \\
\hline SLL & $-1.41 \mathrm{~dB}$ & $-4.4 \mathrm{~dB}$ & $-1.94 \mathrm{~dB}$ & $-4 \mathrm{~dB}$ \\
\hline \multicolumn{5}{|c|}{ Green's function of the homogeneous coupling medium with inside the brain phantom } \\
\hline & Raw data at $1.5-1.75 \mathrm{GHz}$ & TG data at $1.5-1.75 \mathrm{GHz}$ & De-emb. data at $1.5-1.75 \mathrm{GHz}$ & TG de-emb. data at $1.5-1.75 \mathrm{GHz}$ \\
\hline Dim & 1.17 & 0.53 & 1.08 & 0.58 \\
\hline Loc & $2.5 \mathrm{~mm}$ & $1.8 \mathrm{~mm}$ & $1.8 \mathrm{~mm}$ & $1.5 \mathrm{~mm}$ \\
\hline SLL & $-0.5 \mathrm{~dB}$ & $-4.44 \mathrm{~dB}$ & $-1.83 \mathrm{~dB}$ & $-4.73 \mathrm{~dB}$ \\
\hline
\end{tabular}

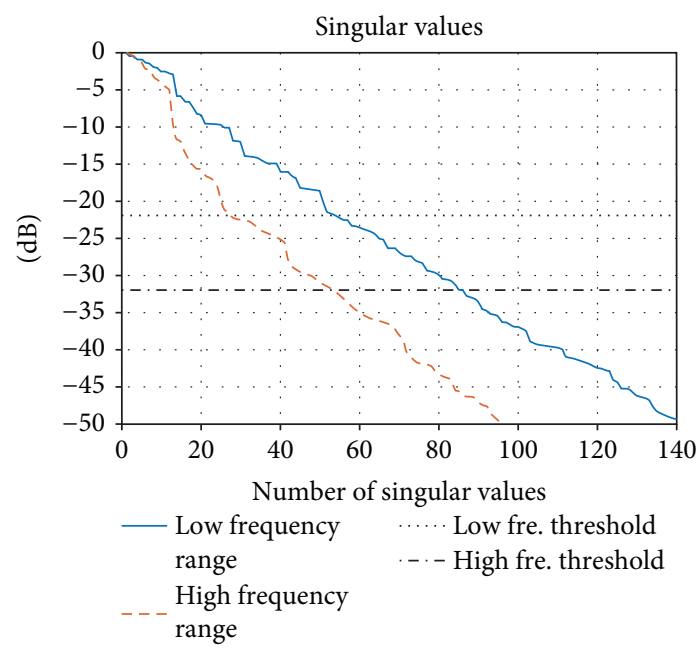

FIGURE 15: Singular value behavior of the scattering operator for low-frequency range (solid blue line) and high-frequency range (dashed red line).

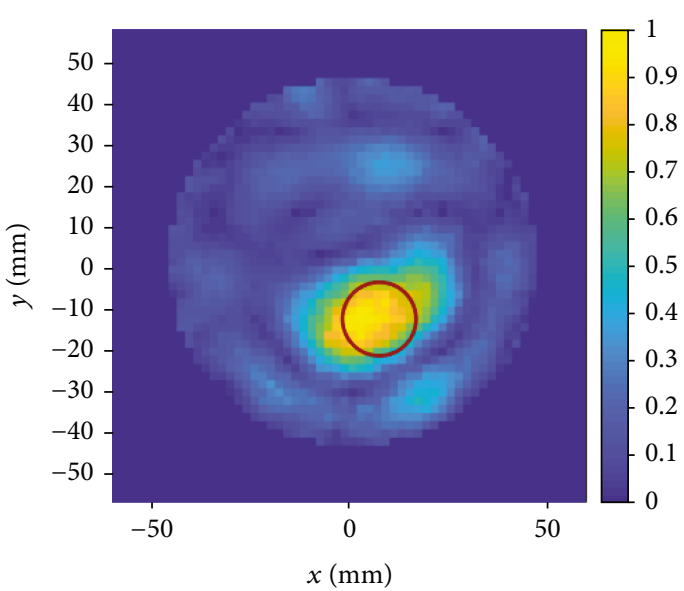

Figure 16: Reconstruction image for Case 2: $1.8 \mathrm{~cm}$ cylinder, raw data with time gating, 1.5 and $1.75 \mathrm{GHz}$, Green's function of the homogeneous coupling medium, and $\tau=-32 \mathrm{~dB}$.

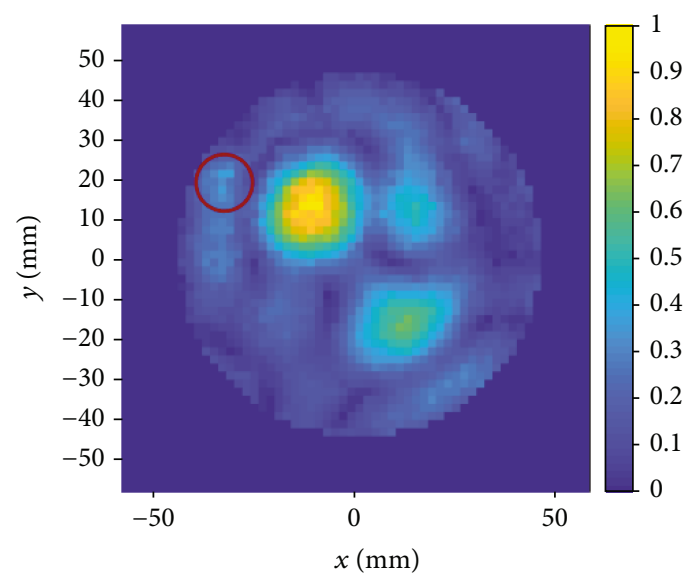

FIgURE 17: Reconstruction image for Case 3: $1.4 \mathrm{~cm}$ cylinder in the outer position, raw data with time gating, 1.5 and $1.75 \mathrm{GHz}$, Green's function of the homogeneous coupling medium, and $\tau=-32 \mathrm{~dB}$.

TABle 2: Cases 2 and 3: evaluated metrics.

\begin{tabular}{lcc}
\hline \multicolumn{2}{c}{ Green's function of the coupling medium, TG data, at $1.5-1.75 \mathrm{GHz}$} \\
& Case 2 & Case 3 \\
\hline Dim & 1.41 & 1.39 \\
Loc & $0.5 \mathrm{~mm}$ & $38 \mathrm{~mm}$ \\
SLL & $-8.4 \mathrm{~dB}$ & $-4.44 \mathrm{~dB}$ \\
\hline
\end{tabular}

parameters measured for adjacent antennas have been enforced to zero (raw data). Moreover, a deembedding operation, allowing at shifting the reference planes directly at the antenna ports, and a time gating, allowing at mitigating the multiple reflections of the signal inside the system, have been alternatively or jointly applied to the raw data. All these different kinds of processed measured data have been analyzed, and the reconstructions have been compared to each other.

The ongoing work concerns the realization and characterization of a 3D and clinically usable version of the MWT system, where the antennas are mounted on a conformable 
helmet adapted to the patient head and of a $3 \mathrm{D}$ realistic phantom of the head that will be used to experimentally test the system before proceeding with the preclinical assessment on patients.

\section{Data Availability}

The data used to support the findings of this study are available from the corresponding author upon request.

\section{Conflicts of Interest}

The authors declare that there is no conflict of interests regarding the publication of this paper.

\section{Acknowledgments}

This work was supported by the Italian Ministry of University and Research (MIUR) under the PRIN project "MiBraScan-Microwave Brain Scanner for Cerebrovascular Diseases Monitoring". The authors would like to acknowledge the valuable help of Eng. G. Dassano for the realization of the switching matrix and of the antenna supports.

\section{References}

[1] R. C. Conceição, J. J. Mohr, and M. O'Halloran, An Introduction to Microwave Imaging for Breast Cancer Detection, Springer International Publishing, 2016.

[2] L. Crocco, Ed.I. Karanasiou, M. L. James, and R. C. Conceição, Emerging Electromagnetic Technologies for Brain Diseases Diagnostics, Monitoring and Therapy, L. Crocco, Ed., Springer International Publishing, 2018.

[3] M. Persson, A. Fhager, H. D. Trefna et al., "Microwave-based stroke diagnosis making global prehospital thrombolytic treatment possible," IEEE Transactions on Biomedical Engineering, vol. 61, no. 11, pp. 2806-2817, 2014.

[4] A. Fhager, S. Candefjord, M. Elam, and M. Persson, "Microwave diagnostics ahead: saving time and the lives of trauma and stroke patients," IEEE Microwave Magazine, vol. 19, no. 3, pp. 78-90, 2018.

[5] M. Hopfer, R. Planas, A. Hamidipour, T. Henriksson, and S. Semenov, "Electromagnetic tomography for detection, differentiation, and monitoring of brain stroke: a virtual data and human head phantom study," IEEE Antennas and Propagation Magazine, vol. 59, no. 5, pp. 86-97, 2017.

[6] A. T. Mobashsher, K. S. Bialkowski, A. M. Abbosh, and S. Crozier, "Design and experimental evaluation of a noninvasive microwave head imaging system for intracranial haemorrhage detection," PLoS One, vol. 11, no. 4, article e0152351, 2016.

[7] M. Maffongelli, S. Poretti, A. Salvade et al., "Design and experimental test of a microwave system for quantitative biomedical imaging," in 2018 IEEE International Symposium on Medical Measurements and Applications (MeMeA), Rome, Italy, June 2018.

[8] Z. Miao, P. Kosmas, and S. Ahsan, "Impact of information loss on reconstruction quality in microwave tomography for medical imaging," Diagnostics, vol. 8, no. 3, p. 52, 2018.

[9] A. Afsari, A. M. Abbosh, and Y. Rahmat-Samii, "A rapid medical microwave tomography based on partial differential equations," IEEE Transactions on Antennas and Propagation, vol. 66, no. 10, pp. 5521-5535, 2018.

[10] A. Afsari, A. M. Abbosh, and Y. Rahmat-Samii, "Modified born iterative method in medical electromagnetic tomography using magnetic field fluctuation contrast source operator," IEEE Transactions on Microwave Theory and Techniques, vol. 67, no. 1, pp. 454-463, 2019.

[11] R. Scapaticci, O. M. Bucci, I. Catapano, and L. Crocco, "Differential microwave imaging for brain stroke followup," International Journal of Antennas and Propagation, vol. 2014, Article ID 312528, 11 pages, 2014.

[12] A. Cavallini, G. Micieli, S. Marcheselli, and S. Quaglini, "Role of monitoring in management of acute ischemic stroke patients," Stroke, vol. 34, no. 11, pp. 2599-2603, 2003.

[13] A. Rocco, M. Pasquini, E. Cecconi et al., "Monitoring after the acute stage of stroke: a prospective study," Stroke, vol. 38, no. 4, pp. 1225-1228, 2007.

[14] J. Gubbi, A. S. Rao, K. Fang, B. Yan, and M. Palaniswami, "Motor recovery monitoring using acceleration measurements in post acute stroke patients," Biomedical Engineering Online, vol. 12, no. 1, p. 33, 2013.

[15] R. Scapaticci, J. Tobon, G. Bellizzi, F. Vipiana, and L. Crocco, "Design and numerical characterization of a low-complexity microwave device for brain stroke monitoring," IEEE Transactions on Antennas and Propagation, vol. 66, no. 12, pp. 73287338, 2018.

[16] J. A. Tobon Vasquez, R. Scapaticci, G. Turvani et al., "First experimental assessment of a microwave imaging prototype for cerebrovascular diseases monitoring," in 2018 International Conference on Electromagnetics in Advanced Applications (ICEAA), Verona, Italy, September 2018.

[17] J. A. Tobon Vasquez, G. Turvani, G. Dassano et al., "Ongoing developments towards the realization of a microwave device for brain stroke monitoring," in 2018 IEEE International Symposium on Antennas and Propagation \& USNC/URSI National Radio Science Meeting, Boston, MA, USA, July 2018.

[18] M. Bertero and P. Boccacci, Introduction to Inverse Problems in Imaging, Institute of Physics, Bristol, U.K., 1998.

[19] I. Sarwar, G. Turvani, M. Casu et al., "Low-cost low-power acceleration of a microwave imaging algorithm for brain stroke monitoring," Journal of Low Power Electronics and Applications, vol. 8, no. 4, p. 43, 2018.

[20] R. Scapaticci, M. Bjelogrlic, J. A. Tobon Vasquez, F. Vipiana, M. Mattes, and L. Crocco, "Microwave technology for brain imaging and monitoring: physical foundations, potential and limitations," in Emerging Electromagnetic Technologies for Brain Diseases Diagnostics, Monitoring and Therapypp. 7-35, Springer, Cham.

[21] R. Scapaticci, L. di Donato, I. Catapano, and L. Crocco, "A feasibility study on microwave imaging for brain stroke monitoring," Progress In Electromagnetics Research B, vol. 40, pp. 305-324, 2012.

[22] N. Joachimowicz, B. Duchene, C. Conessa, and O. Meyer, "Reference phantoms for microwave imaging," in 2017 11th European Conference on Antennas and Propagation (EUCAP), pp. 2719-2722, Paris, France, March 2017.

[23] N. Joachimowicz, C. Conessa, T. Henriksson, and B. Duchene, "Breast phantoms for microwave imaging," IEEE Antennas and Wireless Propagation Letters, vol. 13, pp. 1333-1336, 2014.

[24] S. Romeo, L. di Donato, O. M. Bucci et al., "Dielectric characterization study of liquid-based materials for mimicking breast 
tissues," Microwave and Optical Technology Letters, vol. 53, no. 6, pp. 1276-1280, 2011.

[25] P. M. Meaney, F. Shubitidze, M. W. Fanning, M. Kmiec, N. R. Epstein, and K. D. Paulsen, "Surface wave multipath signals in near-field microwave imaging," International Journal of Biomedical Imaging, vol. 2012, Article ID 697253, 11 pages, 2012.

[26] O. M. Bucci, L. Crocco, R. Scapaticci, and G. Bellizzi, "On the design of phased arrays for medical applications," Proceedings of the IEEE, vol. 104, no. 3, pp. 633-648, 2016.

[27] O. M. Bucci, L. Crocco, and T. Isernia, "Improving the reconstruction capabilities in inverse scattering problems by exploitation of close-proximity setups," Journal of the Optical Society of America. A, vol. 16, no. 7, pp. 1788-1798, 1999.

[28] N. Joachimowicz, B. Duchene, J. A. Tobon Vasquez et al., "Head phantoms for a microwave imaging system dedicated to cerebrovascular disease monitoring," in 2018 IEEE Conference on Antenna Measurements \& Applications (CAMA), Västerås, Sweden, September 2018.

[29] D. M. Pozar, Microwave Engineering, John Wiley and Sons Inc, second edition edition, 1998. 


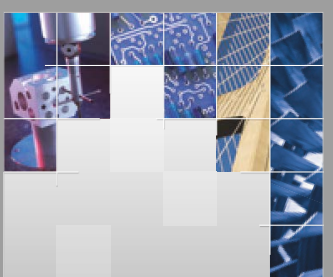

\section{Enfincering}
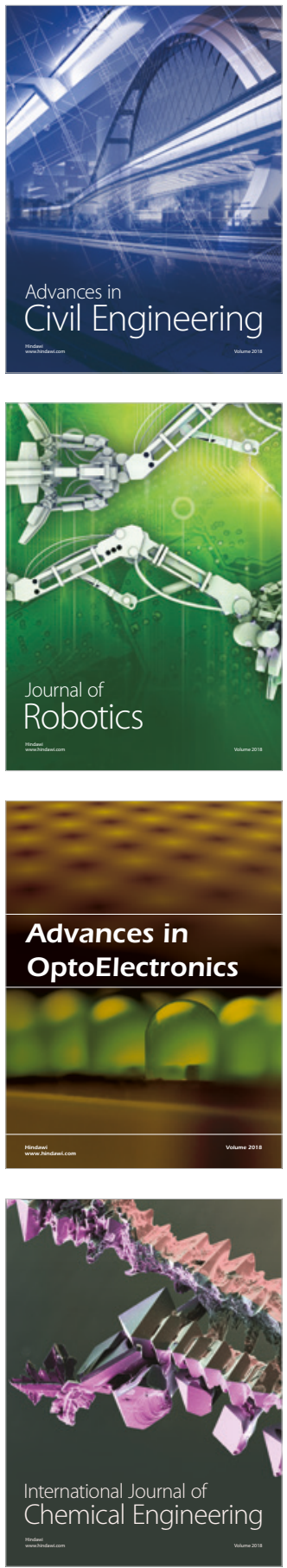

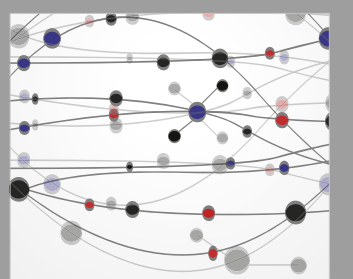

\section{Rotating \\ Machinery}

The Scientific World Journal

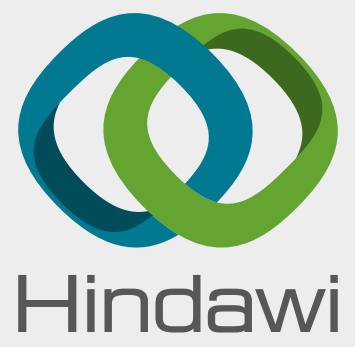

Submit your manuscripts at

www.hindawi.com
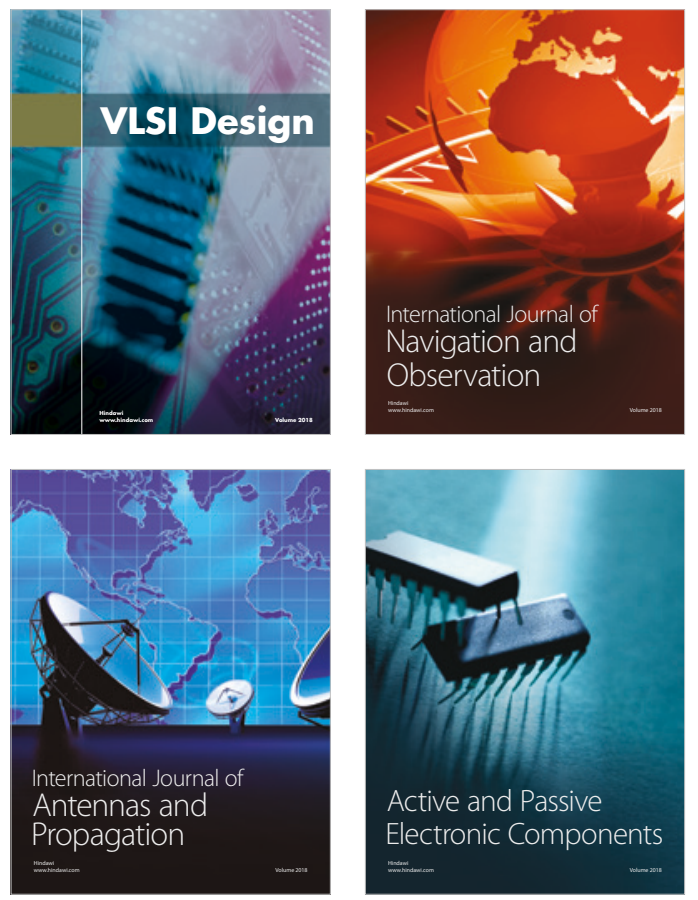
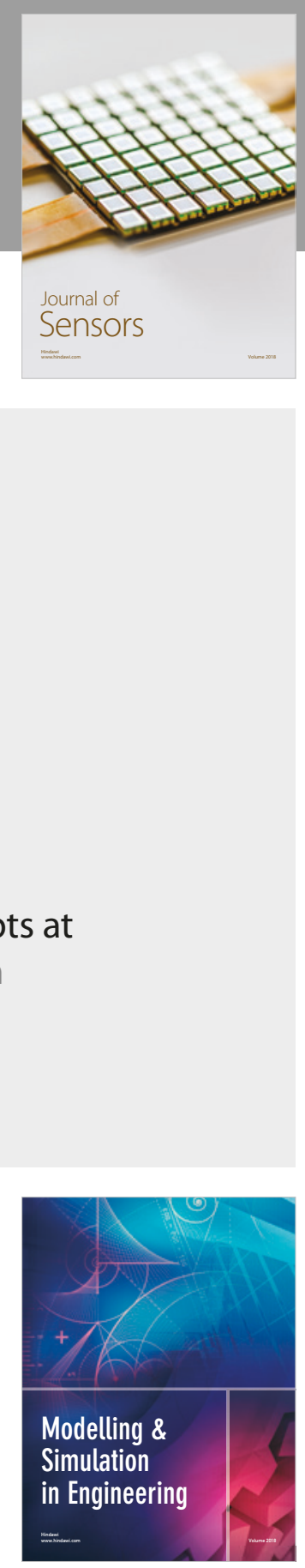

\section{Advances \\ Multimedia}
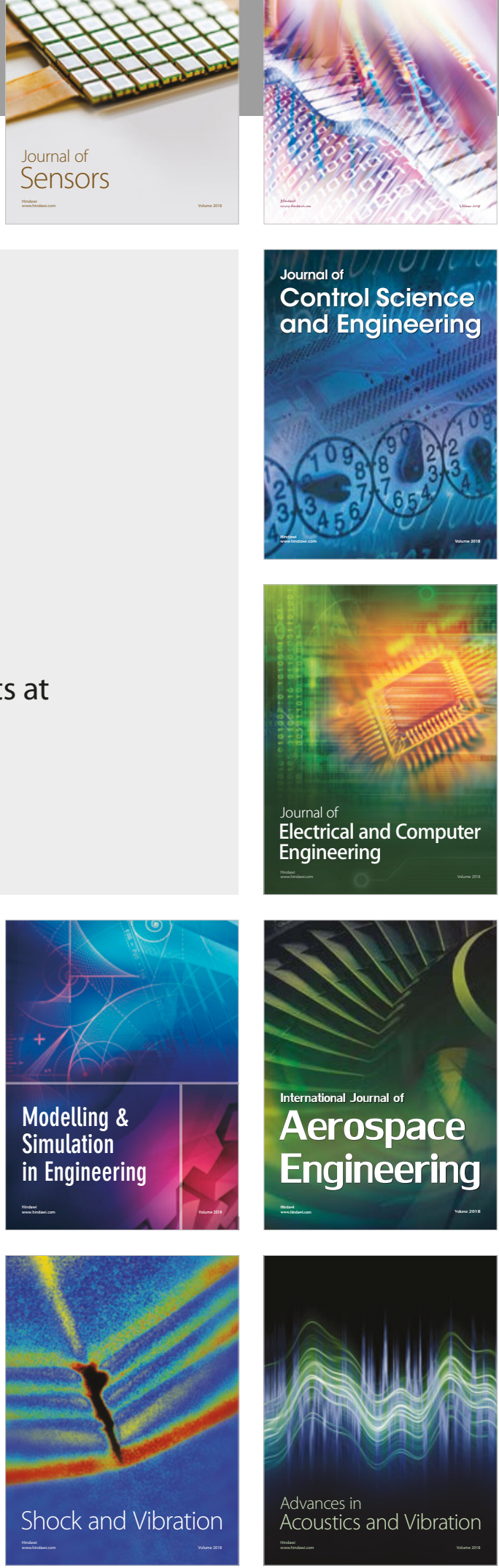